\title{
A 10-Year Follow-Up of Different Intra-Radicular Retainers in Teeth Restored with Zirconia Crowns
}

This article was published in the following Dove Press journal:

Clinical, Cosmetic and Investigational Dentistry

\author{
Debora E Calabro' \\ Alberto N Kojima ${ }^{2}$ \\ Vanessa Gallego Arias \\ Pecorari (iD ${ }^{3}$ \\ Cintia Helena Coury \\ Saraceni (iD) ${ }^{3}$ \\ Markus B Blatz (iD ${ }^{4}$ \\ Mutlu Özcan ${ }^{5}$ \\ Alfredo Mikail Melo \\ Mesquita (i) \\ 'Department of Prosthodontics, Paulista \\ University (UNIP) São Paulo, São Paulo, \\ Brazil; ${ }^{2}$ Department of Dental Materials and \\ Prosthodontics, Paulista State University \\ (UNESP) São José, São Paulo, Brazil; \\ ${ }^{3}$ Department of Dental Materials, Paulista \\ University (UNIP), São Paulo, Brazil; ${ }^{4}$ Unit \\ Center for Dental and Oral Medicine Clinic \\ for Fixed and Removable Prosthodontics \\ and Dental Material Science, University of \\ Zurich, Zurich, Switzerland; ${ }^{5}$ Professor, \\ Department of Preventive and Restorative \\ Sciencie, University of Pennsylvania, \\ Philadelphia, PA, USA
}

Purpose: To evaluate the survival rate in restored teeth with three different types of retainers prior to the fixing of crowns with zirconia through this retrospective clinical study. It is unclear how the type of post and core rehabilitation, and type of resin cement affect the longevity of teeth restored with crowns.

Methods: In a private clinic, a total of 101 retainers installed by the same professional between June 2008 and January 2018, with an average time of 58.2 months (4.8 years), were analyzed regarding the following factors: survival, cement and failure type. Three types of retainers were used according to the indications found in the literature: filling with Z250 light-cured composite resin, 22 elements; fiberglass post with Z250 light-cured composite resin, 45 elements; and cast metallic core in silver-tin alloy, 34 elements. The retainers were cemented with chemically cured cement, U100, U200, or Panavia F.

Results: Data were subjected to Kaplan-Meier analysis $(\mathrm{p}=0.495)$. Although the study presented several limitations, no significant differences were observed in the success rates between the types of intra-radicular retainers and the type of cement. The success rates were as follows: metal core, $97.1 \%$; fiberglass post, $95.6 \%$; and filling, $100 \%$. On average, failures occurred at 48.4 months.

Conclusion: In view of the results, it is possible to conclude that the different retainers evaluated have similar survival rates.

Keywords: fiberpost, type of retainer, cast metallic core, filling core, survival rate

\section{Clinical Implications}

There are several retainers for single crowns; however, there is a wide discussion about their success rate. Different retainers may present similar success rates when properly indicated and prepared.

\section{Introduction}

Caries, fractures, invasive endodontic access, iatrogenies, trauma, non-carious lesions, and extensive restorations can generate great coronary loss to a dental element. Teeth with severe damage may require prosthetic restorations by intraradicular retainers when the remnant tooth structure is no longer sufficient, ${ }^{1-7}$ especially when the coronal destruction involves greater than $50 \%$ of the structure. ${ }^{8}$

Intracanal retainers can be made of different materials: metal (prefabricated or casted), prefabricated fiberglass, or ceramic. ${ }^{9-11}$ However, regardless of the material, the intracanal retainer does not strengthen the remnant dental structure; it confers a restoration retention function only. ${ }^{2}$ Thus, retainers that have properties
Correspondence: Debora E Calabro Department of Prosthodontics, Paulista University, Rua Diogo Jacome, 50 Moema, São Paulo CEP 045 I2-000, Brazil Email deboracalabro@doctor.com 
similar to those of dental structures can reduce the stress generated around $\mathrm{it}^{12}$ and minimize the risk of root fracture.

The selection of the intra-radicular retainer system to be used is a complex procedure, and the quantity and quality of the coronal remnant, the occlusion of the patient, and the type of restoration to be performed should be taken into account. ${ }^{13}$

The cast metallic retainer requires molding of the root cavity, which can be performed with acrylic resin or additional silicone materials, a process that takes a longer time and makes the procedure more expensive. This procedure can be performed using several alloys, such as gold ( $\mathrm{Au})$, titanium (Ti), nickel-chromium (Ni-Cr), and others. ${ }^{14}$

In contrast, the prefabricated post can be made usually in a single consultation if it is resin-reinforced fiberglass, and carbon, glass, quartz, or polyethylene fibers can be incorporated into a resin matrix. ${ }^{14}$

The fiberglass posts are white or transparent and are therefore advantageous when the aesthetics of crowns in vitreous ceramics with high translucency are dependent on the color of the substrate. ${ }^{15}$ However, the lack of remnant structure challenges the use of this system, which is indicated when there is 1.5 to $2.0 \mathrm{~mm}$ of remnant coronary structure. $^{16,17}$

In addition to the coloration, the fiber post also has an elastic modulus that is more similar to that of the dentin. ${ }^{16,18}$ This mechanical property, closer to that of dentinal structures, generates a uniform distribution of stress at the root, reducing the risk of catastrophic failures. ${ }^{19,20}$ The very high elastic modulus can cause excessive stress concentrated around the apex, ${ }^{21}$ which can cause catastrophic failure. ${ }^{22-24}$ Catastrophic failure generates loss of the dental element, while noncatastrophic failure allows repair or exchange of the retainer. The type of failure may be due to the different properties of each retainer. ${ }^{8}$

Nevertheless, other factors can also cause retainer failures: lack of marginal adaptation, failure in the adhesive technique, wedge effect of the cast metallic cores, and failure to indicate the retainer. In addition, oral fluids, bacterial toxins, and other chemical agents that penetrate the tooth-restoration interface may generate discoloration, secondary caries, and marginal microfracture, which may cause fractures or lead to loosening of the retainer. ${ }^{25-28}$

Y-TZP zirconia is a crystalline ceramic that presents a high degree of opacity, which allows the restoration of different substrates and the neutralization of darkened ones, such as cast metallic cores or darkened tooth remnants. ${ }^{29}$

The selection of the cementing agent used was based on the fact that it is important to optimize retention, prevent microleakage, and increase fracture resistance. ${ }^{30}$ The quality of intra-radicular adhesion is influenced by the density and orientation of the dentinal tubules in the different thirds of the root. ${ }^{31}$ In addition to this factor, chemically cured cement does not depend on light-curing.

The aim of this retrospective clinical study was to evaluate the success rates in teeth restored with three different retainer types used prior to fixing of single crowns with zirconia infrastructure over an average period of 58.2 months. The following factors were analyzed: survival, cement, and type of failure.

The null hypothesis was that there would be no significant difference as long as the clinical procedures were performed according to the indications of the literature.

\section{Method}

This study was conducted according to the Helsinki Declaration, revised in 2008, and was approved by the Ethics and Research Committee (CAAE: 22942713.3.00 00.0077) of the São Paulo State University - UNESP, São José dos Campos. São Paulo Brazil. In the medical records of the patients analyzed, there was consent to the application of functional and aesthetic data with research and teaching.

From a total of 1627 medical records of patients who received dental treatment by a single dentist between June 2008 and January 2018 in a private clinic, 53 medical records were selected, and a total of 101 teeth restored with retainers and single crowns with zirconia infrastructure, from 23 men and 30 women, were analyzed.

The inclusion criteria were the following: all elements should have the antagonist present and in occlusion, and the crown should have more than 2 years of final cementation.

Exclusion criteria were not used: bruxism and drugs with the application of not masking the results according to the materials, and get as close to clinical reality as possible.

Before prosthetic treatment, the patients received periodontal treatment, caries control, endodontic treatment, and occlusal adjustment, when necessary.

Teeth that received intra-radicular fiber posts or cast metallic core retainers had whole roots, prior endodontic treatment with good quality filling, absence of signs or symptomatology, and after removal of old restorations, 
carious and contaminated tissue and unsupported enamel and were unfilled, leaving 3 to $4 \mathrm{~mm}$ of apical sealing. For unfilling, the Gates and Largo drill was used according to the diameter of the root.

The restored teeth had as retainers a fiberglass post associated with composite resin filling, a cast metallic core, or only composite resin filling. The criteria for the selection of the retainer system were based on the literature: the teeth receiving filling core only should contain three or more intact walls; for use of the fiberglass post, the elements should contain 1.5 to $2 \mathrm{~mm}$ of healthy coronal remnant; and for the elements with no coronal remnant or with darkening remnant due to the presence of metallic oxides, which would make adhesion difficult, a cast metallic core was used. ${ }^{16,17}$ Thus, the systems were selected based on the remnants.

The 101 elements analyzed were distributed as follows: 22 restored with composite resin filling, 34 with cast metal core, and 45 with fiberglass post associated with the composite resin filling.

Among the 34 restored with cast metallic core, four were cemented with U100 (U100; 3M ESPE), 23 with U200 (U200; 3M ESPE), and seven with Panavia F (Panavia F; Kuraray).

Of the 45 elements restored with fiber post associated with composite resin filling, nine were cemented with U100 (U100; 3M ESPE), 17 with U200 (U200; 3M ESPE), and 19 elements with Panavia F (Panavia F; Kuraray).

\section{Types of Retainers and Modus Operandi Filling with Composite Resin}

After the removal of caries lesions and old restorations, the following procedures were performed: coronal preparation, decontamination with $2 \%$ chlorhexidine (antiseptic Riohex 2\%, Rioquímica), and selective conditioning with $37 \%$ phosphoric acid (Ultra-etch; Ultradent) for $30 \mathrm{sec}-$ onds in enamel, followed by washing for $1 \mathrm{~min}$ with water/air spray, drying of the cavity with absorbent paper and application of the adhesive (Clearfil SE; Kuraray) according to the manufacturer's protocol, and primer application by smear for 20 seconds, followed by a light jet of air and smear bond application for 30 seconds, again followed by a light jet of air and polymerization for 20 seconds. Then, filling with light-curing composite resin (Z250; 3M ESPE) was performed according to the increment method.

\section{Fiberglass Post Associated with Resin Filling}

The fiber posts (Whitepost; FGM) used were selected based on the diameter of the root, with the post diameter being onethird of the root diameter, using a ruler for selection of the manufacturer's posts. Unfilling was performed with Gates and Largo drills, and the final preparation of the canal was performed using the system's milling cutter.

For U100 and U200 (3M ESPE), the element preparation consisted of decontamination with $0.5 \%$ sodium hypochlorite (Dakin's solution; Asfer) and drying with an absorbent paper cone. Post preparation was performed by decontamination with 37\% phosphoric acid (Ultra-etch; Ultradent), water/air spray washing, drying, and application of silane (Dentsply, Chicago, IL, USA) for 3 mins. The insertion of the cement into the canal was performed with a Centrix syringe (Precision; Maquira), followed by light-cure at a 400- to 480-nm wavelength (Radii-Cal; SDI).

For the Panavia F cement (Panavia F; Kuraray), the element was decontaminated with $2 \%$ chlorhexidine (Antiseptic Riohex 2\%, Rioquímica) and 37\% phosphoric acid (Ultra-etch; Ultradent) applied for 30 seconds, followed by washing with water/air spray for $1 \mathrm{~min}$. After drying with an absorbent paper cone, the system adhesive was applied to the canal and to the coronal part of the element, following the manufacturer's protocol. The preparation of the post consisted of decontamination with 37\% phosphoric acid (Ultra-etch; Ultradent), washing with water/air spray, drying, and application of silane (Silane; Dentsply) for 3 mins. The cement insertion in the canal was performed using a Centrix syringe (Maquira, Paraná, Brazil), followed by light-cure at a 400- to 480-nm wavelength (Radii-Cal; SDI).

After post cementation, composite resin filling was initiated by conditioning the enamel with $37 \%$ phosphoric acid (Ultra-etch; Ultradent), followed by washing for 1 min with water/air spray and cavity drying with absorbent paper. The adhesive (ClearFil SE; Kuraray) was applied according to the manufacturer's instructions, followed by primer application by smear for 20 seconds, followed by a light air jet and bond application by smear for 30 seconds, light air jet again, and light-curing for 20 seconds at 400-480 nm (Radii-Cal; SDI). The restoration with Z250 light-curing composite resin (Z250; 3M ESPE) was then performed using the increment technique.

\section{Retainer: Cast Metallic Core}

The cast metallic cores were prepared using the modeling technique. After preparation of the canal, it was isolated 
with solid petroleum jelly (Biochemistry, São José do Rio Preto, Brazil) and red acrylic resin (Pattern Resin LS; CG), and modeling was performed using a modeling post (Pinjet; Angelus). Then, the mold was sent to the dental laboratory for casting in metal alloy (Silver-Tin; Primalloy) using the lost-wax technique.

The metallic cores were decontaminated with $70 \%$ alcohol and blasted (Microjato Plus, Bio Art) with 50micrometer aluminum oxide (Aluminum Oxide, Bio Art) for 20 seconds at a pressure of 2 bar.

For U100 and U200 (3M ESPE), the element was decontaminated with $0.5 \%$ sodium hypochlorite (Dakin's solution; Asfer) and drying with an absorbent paper cone. The insertion of the cement into the canal was performed with a Centrix syringe (Precision; Maquira), followed by light-curing at $400-480 \mathrm{~nm}$ (Radii-Cal; SDI).

For the Panavia F (Kuraray) cement, the element was decontaminated with $2 \%$ chlorhexidine (antiseptic Riohex 2\%, Rioquímica). Then, 37\% phosphoric acid (Ultra-etch; Ultradent) was applied for 30 seconds, followed by washing with water/air spray for $1 \mathrm{~min}$. After drying with an absorbent paper cone, the system adhesive was applied to the canal and to the coronal part of the element. The insertion of the cement into the canal was performed with a Centrix syringe (Precision; Maquira), followed by light-curing at $400-480 \mathrm{~nm}$ (Radii-Cal; SDI).

After fixing the retainer, all elements were restored with fixed single prosthesis with zirconia coping. Regardless of the retainer, all of the preparations were performed with chamfer finish, drill number 4138 (KG Sorensen), and the finishing of the preparations was performed with a 30-blade multilayer drill bit (Komet) against a 1:5 multiplier angle (T3 Line E 200; Sirona). Between the consultations, temporary crowns in acrylic resin were cemented with temporary cement with calcium hydroxide (Provicol; Voco).

The molds were obtained with the addition of silicone (Futura AD, DFL), using gingival retraction with the retraction cord (Ultrapak; Ultradent) according to the indication of the gingival biotype, using the simultaneous technique.

All crowns were cemented with chemically cured resin cement according to the manufacturer's recommendations. Before cementation, the adaptation was verified with an exploratory probe, and radiography and the adjustment of the occlusion and proximal region was performed. The crown/cement distribution was performed as follows: 14/ U100 (3M ESPE); 54/U200 (3M ESPE); 33/Panavia F (Kuraray).
The survival rate was assessed according to the absence of complications. The complications included loosening of the retainer, fracture of any region of the dental element, fracture of the retainer, secondary caries, pain, need for endodontic treatment after fixing the prosthesis, and patient dissatisfaction. Retention longevity was measured from the month of cementation until January 2018 for the elements that did not fail and until the month of complication for those that failed.

The data obtained were described in tables according to: region, type of retainer, cement used, failures and failure time. Then, Kaplan-Meier survival analysis was used to compare the success rates of the different variables under study as a function of time, using $\alpha=0.05$. The statistical program SPSS 21 (IBM, USA, Chicago, IL) was used.

\section{Results}

One-hundred one cases of teeth with retainers were analyzed for a mean time of 58.24 months, with a standard deviation of 25.72 months; the minimum observed time was 26 months, and the maximum was 115 months.

The types of intra-radicular retainer used in the sample were as follows: $33.7 \%$ (34 teeth) with cast metallic core, $44.6 \%$ (45 teeth) with fiberglass post, and $21.8 \%$ (22 teeth) with filling, totaling 101 teeth.

The distribution of the elements in the arch and in the retainers is represented in the demographic table (Table 1).

The distribution of the retainers according to cement was as follows: for the cast metallic cores, four were cemented with U100, 23 with U200, and seven with Panavia F. For the fiberglass posts, nine were cemented with U100, 17 with U200, and 19 with Panavia F, as shown in Table 2.

As shown in Table 3, of the failure cases, one (33.3\%) had a cast metallic core, and two $(66.7 \%)$ were of fiberglass; no failures were observed in the teeth that used filling as the intra-radicular retainer material.

The failures found included fiberglass post fracture in an element that was cemented with Panavia F, which was restored with a cast metallic core; root fracture in an element that was restored with fiberglass post cemented with Panavia F, which was extracted, and an implant was fixed in the region; and root fracture in a tooth that was cemented with a metallic core bonded with Panavia F, which was also extracted, and an implant was fixed in the region. The three failures presented in the study were in different periods, but all occurred in the same patient. 
Table I Demographic Table

\begin{tabular}{|c|c|c|c|c|c|c|c|c|c|c|c|c|}
\hline & \multicolumn{6}{|c|}{ Mandible } & \multicolumn{6}{|c|}{ Maxilla } \\
\hline Total of teeth & \multicolumn{6}{|l|}{20} & \multicolumn{6}{|l|}{81} \\
\hline \multirow[t]{2}{*}{ Region } & \multicolumn{3}{|c|}{ Anterior } & \multicolumn{3}{|c|}{ Posterior } & \multicolumn{3}{|c|}{ Anterior } & \multicolumn{3}{|c|}{ Posterior } \\
\hline & \multicolumn{3}{|l|}{4} & \multicolumn{3}{|l|}{16} & \multicolumn{3}{|l|}{46} & \multicolumn{3}{|l|}{35} \\
\hline \multirow[t]{2}{*}{ Retainer } & FP & CM & FC & FP & CM & FC & FP & CM & FC & FP & CM & FC \\
\hline & 4 & 0 & 0 & 7 & 6 & 3 & 19 & 12 & 15 & 15 & 16 & 4 \\
\hline
\end{tabular}

Abbreviations: FP, Fiberglass post; CM, Cast metallic core; FC, Filling core.

Table 2 Division of Retainers According to the Cement Used

\begin{tabular}{|l|l|l|l|}
\hline & U100 & U200 & Panavia F \\
\hline Metallic Core & 4 & 23 & 7 \\
Fiberglass Post & 9 & 17 & 19 \\
\hline
\end{tabular}

Table 3 Summary of Case Processing

\begin{tabular}{|l|l|l|l|l|}
\hline Post Group & \multirow{2}{*}{ Total No. } & \multirow{2}{*}{ No. of Events } & \multicolumn{2}{|l|}{ Censored } \\
\cline { 4 - 5 } & & & No. & Percentage \\
\hline Metallic core & 34 & 1 & 33 & $97.1 \%$ \\
Fiberglass & 45 & 2 & 43 & $95.6 \%$ \\
Filling & 22 & 0 & 22 & $100.0 \%$ \\
Global & 101 & 3 & 98 & $97.0 \%$ \\
\hline
\end{tabular}

No significant difference was observed between the intra-radicular retainers analyzed $(\mathrm{p}=0.495)$.

The survival rates as a function of time for the types of retainers are shown in Graph 1. The metallic core survival rate was $97.1 \%$, that of the fiberglass post was $95.6 \%$, and that of the filling was $100 \%$, as shown in Kaplan-Meier Graph 1 (Figure 1).

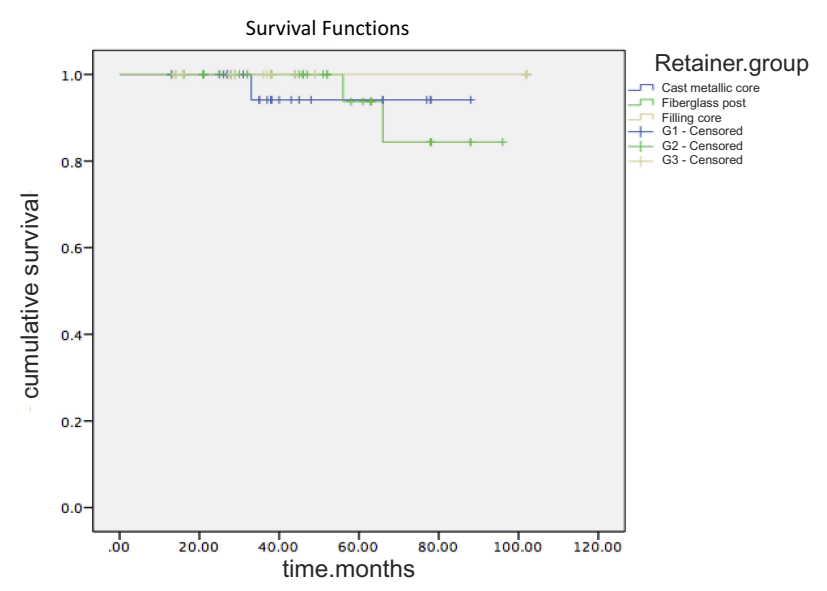

Figure I Graph - survival functions.
Of the cases that presented failures, the mean was 48.4 months, with a standard deviation of 16.38 months. In addition to the failures with the retainers, the present study presented two other failures related to the crowns, with one cementation failure in a crown cemented with U100 on a cast metallic core, which was re-cemented with U200, and the other a fracture of the chipping recoating ceramics in a cemented crown on a fiberglass post with Panavia F, which was restored. All failures are presented in Table 4 as a function of time.

\section{Discussion}

The aim of this study was to clinically evaluate the survival rates of three types of fixed prosthesis retainers: composite resin filling, fiberglass post, and cast metallic core. In view of the results, the null hypothesis that there would be no significant difference between them was accepted. This hypothesis was demonstrated based on the indications for each retainer found in the literature.

All elements of this study were restored with bilayer zirconia crowns, ie, zirconia infrastructure with the application of aesthetic coating ceramics. Zirconia, besides having a flexural strength of $800 \mathrm{MPa}$ at $1200 \mathrm{MPa}$ and a fracture

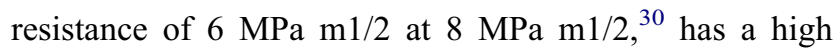
degree of opacity because it is a polycrystalline ceramic, which indicates its use on a darkened substrate restoration,

Table 4 Time, in Months, and Cause of the Failures That Occurred

\begin{tabular}{|l|l|}
\hline Failure Cause & Failure Time (Months) \\
\hline Cementation & 29 \\
Post & 56 \\
Post & 66 \\
Post & 33 \\
Crown & 58 \\
Failure mean (SD) & $48.40(16.38)$ \\
\hline
\end{tabular}


as a cast metallic core; thus, it presents versatility over different retainers and ensures the final aesthetics of the restoration.

The use of chemically cured cements is possible with different types of retainers, as the cements do not depend on light for their polymerization. Thus, the difficult access of light in the apical third of the root is not a negative factor because different densities and orientations of the dentinal tubules already exist in the different thirds of the root, ${ }^{31}$ and the resin cements present higher retention than zinc phosphate cement. ${ }^{32}$

Several biological, mechanical, and aesthetic factors are involved in the survival rate of the restoration, and the selection of the retainer must satisfy and optimize these factors. ${ }^{33}$ The selection of the type of retainer in this study was determined from the coronal remnant of the element to be restored with the fixed prosthesis. The indication for a fiber post was the presence of dentinal remnant with a minimum of 1.5 to $2.0 \mathrm{~mm}$ height, with no metallic oxides present; thus, cast metallic cores were performed in elements that had remnants smaller than 1.5 to $2.0 \mathrm{~mm}$; in the cases where filling was performed, the dental element had at least three walls, as indicated in the literature. ${ }^{17}$ The lack of coronal remnant is cited as one of the challenges for the use of fiberglass posts; another study also recommended the criterion of 1.5 to $2 \mathrm{~mm}$ of remnant coronal structure. ${ }^{16}$

In the present study, three types of retainers for fixed prosthesis were used: an intra-radicular retainer with a fiberglass post, a cast metallic core intra-radicular retainer, and a resin filling retainer and/or dental remnant only. There were no significant differences in the survival rates between them. Another clinical study with a 12-month follow-up also stated that different retainers, including fiberglass posts and cast metallic core systems, have high success rates. ${ }^{34}$ Although there was no significant difference between fiberglass and metal retainers, another study, ${ }^{35}$ with a sample of 40 elements, in a 6-month follow-up, divided into two groups, fiberglass post and metallic core, concluded that fiberglass posts have better clinical performance than metallic cores. In another study, ${ }^{36}$ with a sample of 203 elements, which compared prefabricated and individualized fiber posts, metallic cores, and filling with no retainer in 1-, 3-, and 5-year follow-up periods, despite the $12.8 \%$ of failures after the last follow-up consultation, showed no significant differences between the groups, allowing the conclusion that all systems used in total restorations of ceramics present similar good clinical performance.
The results of the present study revealed three cases of failure: one with a cast metallic core where root fracture occurred 33 months after crown cementation; and two with fiberglass, one with a fracture of the post 66 months after cementation, and the other with root fracture at the fiber post after 55 months. No cases of failure were observed in teeth that used filling as the intra-radicular retainer material. In spite of the absolute numbers, the statistical tests did not show significant differences between the groups, and the literature review ${ }^{33}$ indicated that fiberglass posts have presented similar performance to cast metallic posts. In addition to survival, the literature reviews described possible types of failure and their prevalence in each system: in cast metallic cores, fracture of the retainer, loss of retention of the retainer, and/or crown and root fracture occur, while in fiberglass posts, the most common failure is loss of retention. ${ }^{37}$ Thus, metallic retainer failures are mostly catastrophic, unlike with fiberglass posts. ${ }^{33}$

Like the literature reviews, laboratory and finite element studies have also cited the differences between failures in retainer systems and have shown that the greatest numbers of non-catastrophic failures are in fiberglass posts when compared with cast metallic cores, justifying the results where minor stresses are generated at the root when the fiberglass post is used, reducing the chance of the occurrence of root fracture, different from the higher fracture rates with cast metallic cores. ${ }^{15,38,39}$

These results of lower catastrophic failure rates for the fiberglass post are associated with the lower elastic modulus, as the fiberglass posts are more similar to dental structures than are metal retainers. ${ }^{39}$ This elastic modulus, which is similar to that of dentin, generates a better load distribution throughout the system; therefore, the fracture resistance is smaller than in metallic cores, ${ }^{16}$ and the fiberglass post shows a more favorable biomechanical behavior. $^{40}$

Although the three groups of retainers showed no significant differences and the fiberglass post showed an elastic modulus that was more similar to that of dentin structures than the cast metallic core, the group in which a composite resin filling core was used was the only one that did not show any failures. In another 5-year clinical follow-up study comparing metallic core, fiberglass post, and filling core, there were also no differences among the groups. ${ }^{36}$ Another study compared composite resin filling core and fiberglass post, showing that the insertion of the intra-radicular retainer does not reduce the risk of failure of the filling core. ${ }^{41}$ 
Considering that the filling core was the only type that did not show failures and still maintained the largest amount of tooth structure, and because it is a less complex procedure, whenever possible, it is more appropriate to perform this procedure, depending on the quantity and the quality of the remnant. The prosthesis will present an equal or better clinical performance.

In the present study, despite no significant differences identified, all failures occurred in the same patient: two root fractures, one with a fiberglass post, element 12 , and one with a cast metallic core, element 22 , and a fracture of the intraradicular retainer, fiberglass post, element 15 . All of them had been cemented with Panavia F. During the onset of failures, the patient reported having started using the psychotropic fluoxetine, which may induce parafunctional activity. ${ }^{42}$

The results of this study showed that there were no differences among the retainers in a clinical follow-up with a mean time of 58.2 months (4.8 years), where time, cement, and type of failure were evaluated. These results provide the dental surgeon with the possibility of system selection, including resin filling in vital and nonvital teeth, fiberglass post, or cast metallic core, according to the indications and following the clinical protocol of each system.

\section{Conclusion}

In view of the results, one can conclude that the different retainers evaluated have similar survival rates, with excellent clinical performance, conditioned to the adequate indications of each system. Thus, in this clinical study and laboratory protocol, the different retainers were determined to be accessible and viable prosthetic solutions for dental surgeons.

\section{Disclosure}

The authors report no conflicts of interest in this work.

\section{References}

1. Shillingburg HT, Sather DA, Wilson EL, et al. Fundamentals of Fixed Prosthodontics. 4th ed. Hanover Park: Quintessence; 2012:214, 132.

2. Kimmel SS. Restoration of endodontically treated tooth containing wide or flared canal. N Y State Dent J. 2000;66:36-40.

3. Kalay TS, Yildirim T, Ulker M. Effects of different cusp coverage restorations on the fracture resistance of endodontically treated maxillary premolars. J Prosthet Dent. 2016;116:404-410. doi:10.1016/j. prosdent.2016.02.007

4. Ibrahim AMB, Richards LC, Berekally TL. Effect of remnant tooth structure on the fracture resistance of endodontically-treated maxillary premolars: an in vitro study. J Prosthet Dent. 2016;115:290-295. doi:10.1016/j.prosdent.2015.08.013
5. Amarante MV, Pereira MV, Darwish FA, Camarão AF. Stress prediction in a central incisor with intra-radicular restorations. Mater Res. 2011;14:189-194. doi:10.1590/S1516-14392011005000025

6. Pierrisnard L, Bohin F, Renault P. Corono-radicular reconstruction of pulpless teeth: a mechanical study using finite element analysis. J Prosthet Dent. 2002;88:442-448. doi:10.1067/mpr.2002.128376

7. Tang W, Wu Y, Smales RJ. Identifying and reducing risks for potential fractures in endodontically treated teeth. $J$ Endod. 2010;36:609-617. doi:10.1016/j.joen.2009.12.002

8. Figueiredo FE, Martins-Filho PR, Faria-e-Silva AL. Do metal post-retained restorations result in more root fractures than fiber post-retained restorations? A systematic review and meta-analysis. J Endod. 2015;41:309-316. doi:10.1016/j.joen.2014.10.006

9. Veríssimo C, Júnior PCS, Soares CJ, Noritomi PY, Santos-Filho PC. Effect of the crown, post, and remnant coronal dentin on the biomechanical behavior of endodontically treated maxillary central incisors. J Prosthet Dent. 2014;111:234-246. doi:10.1016/j.prosdent.2013.07.006

10. Barbizam JV, White SN. Fatigue susceptibility of an endodontic fibre post material. Int Endod J. 2014;47:202-209. doi:10.1111/ iej.2014.47.issue-2

11. Lassila LV, Tanner J, Le Bell AM, et al. Flexural properties of fiber reinforced root canal posts. Dent Mater. 2004;20:29-36. doi:10.1016/ S0109-5641(03)00065-4

12. Mahmoudi M, Saidi AR, Amini P, Hashemipour MA. Influence of inhomogeneous dental posts on stress distribution in tooth root and interfaces: three-dimensional finite element analysis. $J$ Prosthet Dent. 2017;391:742-751.

13. Naumann M, Blankenstein F, Kiessling S, Dietrich T. Risk factors for failure of glass fiber-reinforced composite post restorations: a prospective observational clinical study. Eur J Oral Sci. 2005;113:519-524. doi:10.1111/eos.2005.113.issue-6

14. Pegoretti A, Fambri L, Zappini G, Bianchetti M. Finite element analysis of a glass fibre reinforced composite endodontic post. Biomaterials. 2002;23:2667-2682. doi:10.1016/S0142-9612(01) 00407-0

15. Maroli A, Hoelcher KAL, Reginato VF, Spazzin AO, Caldas RA, Bacchi A. Biomechanical behavior of teeth without remnant coronal structure restored with different post designs and materials. Mater $\mathrm{Sci}$ Eng C Mater Biol Appl. 2017;76:839-844. doi:10.1016/j. msec.2017.03.152

16. Al-Omiri MK, Mahmoud AA, Rayyan MR, Abu-Hammad O. Fracture resistance of teeth restored with post-retained restorations: an overview. $J$ Endod. 2010;36:1439-1449. doi:10.1016/j. joen.2010.06.005

17. Monticelli F, Grandini S, Goracci C, Ferrari M. Clinical behavior of translucent-fiber posts: a 2-year prospective study. Int J Prosthodont. 2003;16:593-596.

18. Rosa RA, Barreto MS, Moraes Rdo A, et al. Influence of endodontic sealer composition and time of fiber post cementation on sealer adhesiveness to bovine root dentin. Braz Dent J. 2013;24:241-246. doi:10.1590/0103-6440201302154

19. Coelho CS, Biffi JC, Silva GR, Abrahão A, Campos RE, Soares CJ. Finite element analysis of weakened roots restored with composite resin and posts. Dent Mater J. 2009;28:671-678. doi:10.4012/ dmj.28.671

20. Maroulakos G, Nagy WW, Kontogiorgos ED. Fracture resistance of compromised endodontically treated teeth restored with bonded post and cores: an in vitro study. J Prosthet Dent. 2015;114:390-397. doi:10.1016/j.prosdent.2015.03.017

21. Newman MP, Yaman P, Dennison J, Rafter M, Billy E. Fracture resistance of endodontically treated teeth restored with composite posts. J Prosthet Dent. 2003;89:360-367. doi:10.1067/mpr.2003.75

22. Barjau-Escribano A, Sancho-Bru JL, Forner-Navarro L, RodríguezCervantes PJ, Pérez-Gónzález A, Sánchez-Marín FT. Influence of prefabricated post material on restored teeth: fracture strength and stress distribution. Oper Dent. 2006;31:47-54. doi:10.2341/04-169 
23. Martelli H Jr, Pellizzer EP, Rosa BT, Lopes MB, Gonini A Jr. Fracture resistance of structurally compromised root filled bovine teeth restored with accessory glass fibre posts. Int Endod J. 2008;41:685-692. doi:10.1111/j.1365-2591.2008.01424.x

24. Barcellos RR, Correia DP, Farina AP, Mesquita MF, Ferraz CC, Cecchin D. Fracture resistance of endodontically treated teeth restored with intra-radicular post: the effects of post system and dentine thickness. J Biomech. 2013;46:2572-2577. doi:10.1016/j.jbiomech.2013.08.016

25. Geramipanah F, Rezaei SM, Sichani SF, Sichani BF, Sadighpour L. Microleakage of different post systems and a custom adapted fiber post. J Dent (Tehran). 2013;10:94-102.

26. Duc O, Krejci I. Effects of adhesive composite core systems on adaptation of adhesive post and cores under load. J Dent. 2009;37:622-626. doi:10.1016/j.jdent.2009.04.005

27. Alves J, Walton R, Drake D. Coronal leakage: endotoxin penetration from mixed bacterial communities through obturated, post-prepared root canals. J Endod. 1998;24:587-591. doi:10.1016/S0099-2399(98)80115-5

28. Mannocci F, Ferrari M, Watson TF. Microleakage of endodontically treated teeth restored with fiber posts and composite cores after cyclic loading: a confocal microscopic study. J Prosthet Dent. 2001;284-291. doi:10.1067/mpr.2001.113706

29. Denry I, Kelly JR. State of the art of zirconia for dental applications. Dent Mater. 2008;24:299-307. doi:10.1016/j.dental.2007.05.007

30. Thompson JY, Stoner BR, Piascik JR, Smith R. Adhesion/cementation to zirconia and other non-silicate ceramics: where are we now? Dent Mater. 2011;27:71-82. doi:10.1016/j.dental.2010.10.022

31. Goracci C, Tavares AU, Fabianelli A, et al. The adhesion between fiber posts and root canal walls: comparison between microtensile and push-out bond strength measurements. Eur J Oral Sci. 2004;112:353-361. doi:10.1111/eos.2004.112.issue-4

32. Junge T, Nicholls JI, Phillips KM, Libman WJ. Load fatigue of compromised teeth: a comparison of 3 luting cements. Int J Prosthodont. 1998;11:558-564.

33. Soares CJ, Valdivia AD, da Silva GR, Santana FR, Menezes Mde S. Longitudinal clinical evaluation of post systems: a literature review. Braz Dent J. 2012;23:135-740. doi:10.1590/S0103-64402012000200008
34. Preethi G, Kala M. Clinical evaluation of carbon fiber reinforced carbon endodontic post, glass fiber reinforced post with cast post and core: a one-year comparative clinical study. J Conserv Dent. 2008;11:162-167. doi:10.4103/0972-0707.48841

35. Gbadebo OS, Ajayi DM, Oyekunle OO, Shaba PO. Randomized clinical study comparing metallic and glass fiber post in restoration of endodontically treated teeth. Indian J Dent Res. 2014;25:58-63. doi:10.4103/0970-9290.131126

36. Cloet E, Debels E, Naert I. controlled clinical trial on the outcome of glass fiber composite cores versus wrought posts and cast cores for the restoration of endodontically treated teeth: a 5-year follow-up study. Int J Prosthodont. 2017;30:71-79. doi:10.11607/ijp.4861

37. Marchionatti AME, Wandscher VF, Rippe MP, Kaizer OB, Valandro LF. Clinical performance and failure modes of pulpless teeth restored with posts: a systematic review. Braz Oral Res. 2017;31. doi:10.1590/1807-3107bor-2017.vol31.0064

38. Aggarwal R, Gupta S, Tandan A, Gupta NK, Dwivedi R, Aggarwal R. Comparative evaluation of fracture resistance of various post systems using different luting agents under tangential loading. $J$ Oral Biol Craniofac Res. 2013;3:63-67. doi:10.1016/j. jobcr.2013.04.002

39. Santos AF, Meira JB, Tanaka CB, et al. Can fiber posts increase root stresses and reduce fracture? J Dent Res. 2010;89:587-591. doi:10.1177/0022034510363382

40. Bosso K, Gonini Júnior A, Guiraldo RD, Berger SB, Lopes MB. Stress generated by customized glass fiber posts and other types by photoelastic analysis. Braz Dent J. 2015;26:222-227. doi:10.1590/ 0103-6440201300256

41. Chiba A, Hatayama T, Kainose K, et al. The influence of elastic moduli of core materials on shear stress distributions at the adhesive interface in resin built-up teeth. Dent Mater J. 2017;36:95-102. doi:10.4012/dmj.2016-160

42. Lavigne GJ, Manzine AC. Bruxism. In: Kryger MH, Roth T, Dement WC, editors. Principles and Practice of Sleep Medicine. Philadelphia: W. B. Saunders; 2000:773-785. 


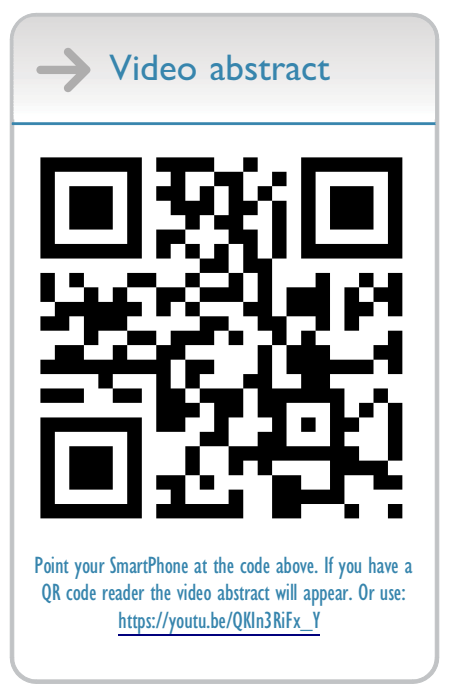

Clinical, Cosmetic and Investigational Dentistry

\section{Publish your work in this journal}

Clinical, Cosmetic and Investigational Dentistry is an international, peer-reviewed, open access, online journal focusing on the latest clinical and experimental research in dentistry with specific emphasis on cosmetic interventions. Innovative developments in dental materials, techniques and devices that improve outcomes and patient satisfaction and preference will be highlighted. The manuscript management system is completely online and includes a very quick and fair peer-review system, which is all easy to use. Visit http://www.dovepress.com/testimonials.php to read real quotes from published authors. 